\title{
Dynamic Fracture of a Semi-Crystalline Bio-Based Polymer Pipe: Effect of Temperature
}

\section{Jean Benoit Kopp, Jeremie Girardot}

Arts et Metiers Institute of Technology, Université de Bordeaux, CNRS, INRA, INP, I2M, HESAM Université, Talence, France

Email: jean-benoit.kopp@ensam.eu

How to cite this paper: Kopp, J.B. and Girardot, J. (2021) Dynamic Fracture of a Semi-Crystalline Bio-Based Polymer Pipe: Effect of Temperature. Journal of Minerals and Materials Characterization and Engineering, 9, 227-244.

https://doi.org/10.4236/jmmce.2021.93016

Received: March 22, 2021

Accepted: May 9, 2021

Published: May 12, 2021

Copyright $\odot 2021$ by author(s) and Scientific Research Publishing Inc. This work is licensed under the Creative Commons Attribution International License (CC BY 4.0).

http://creativecommons.org/licenses/by/4.0/

\begin{abstract}
The influence of temperature on the resistance to rapid crack propagation of a semi-crystalline bio-based polymer was studied. The experimental results described in this study allow to initiate a first discussion on the role of viscosity and its link with the fracture behaviour and a heterogeneous microstructure such as the semi-crysalline polymer. Dynamic fracture tests on pipes were carried out. It would appear that a temperature decrease of approximately $40^{\circ} \mathrm{C}$ relative to ambient has no significant influence on the average crack propagation velocity $\left(\approx 0.6 c_{R}\right)$, fracture energy and surface roughness. On the contrary, crack propagation paths seem to vary with temperature. The difference in fracture behaviour between the amorphous and crystalline phase varies significantly as a function of temperature. The difference between the initiation resistance and the rapid propagation also varies. This difference seems to be significantly reduced by lowering the temperature. The mechanisms of cavitation damage and plastic flow are increasingly limited by the decrease in temperature (and therefore in macromolecular mobility). Crack propagation in the pipe is more extensive and therefore more critical. This is emphasised in particular by the probability of the material to be macro-branched as the temperature decreases.
\end{abstract}

\section{Keywords}

Dynamic Fracture, Rapid Crack Propagation, Semi-Crystalline, Pipe, Fractography

\section{Introduction}

Polyamides and more generally semi-crystalline polymers are widely used for industrial applications [1] [2]. This is evidenced by the number of new grades that have appeared regularly over the last few decades. Their micro-structures 
and intrinsic properties are appreciated for designing parts with structural strength. Polyamides have a fairly good fatigue strength and resistance to repeated impact over a wide range of operating temperatures. The incorporation of reinforcements (such as glass fibres or shock absorbing copolymers) improves certain mechanical properties [3]. However, the mechanical properties of polyamides, particularly in terms of fatigue and fracture, are affected by the effect of humidity.

It is accepted that the mechanical behaviour of a semi-crystalline is viscoelastoplastic ductile [4]. The micro-structure of a semi-crystalline polymers is mainly composed of an amorphous phase where damage and plasticity mechanisms occurs and thus increase the ductility of the material. In contrast to the crystalline phase which is rather considered to be brittle. The material is damaged in the tensile direction by a cavitation mechanism [5] [6] [7] [8]. This mechanism usually occurs in the amorphous phase of the material. Microcracks associated with cavitation have been observed by micro-tomography at relatively low loading levels [5] [9]. The material failure is generated by the coalescence of these microcracks when they reach a critical size [5] [10] [11]. The loading conditions (strain rate in particular), the environment (temperature [12] and humidity [13]) and the degree of crystallinity [14] [15] significantly affect the fracture behaviour of the material. When the strain rate increases and/or the test temperature decreases the amorphous phase and thus the material becomes more brittle. This is also the case when the material is aged [13]. These different conditions can generate abrupt fractures and more precisely rapid crack mechanisms.

The resistance to rapid crack propagation for this type of material has been little published [16] [17], the effect of temperature on fracture behaviour also. It is more common to evaluate the resistance to slow crack propagation for this type of material [18] [19]. The multiphase microstructure (amorphous and crystalline phases) and the sensitivity to the strain rate of the fracture behaviour make it difficult to estimate a material parameter (such as fracture energy) described by the formalism of linear elastic fracture mechanics (LEFM) [16] [17]. Cracking velocity and thus cracking duration have a major influence on the response of the material. The macromolecular mobility is more or less limited depending on the cracking duration. However, this mobility plays a major role in the cracking process. In slow crack regime, cavitation damage and plasticity mechanisms precede crack propagation. They are the source of a large amount of dissipated energy in the volume and thus of the ductility of the material. At short times, cavitation and plasticity do not seem to be able to be activated in favour of rapid cracking. This is referred to as brittle material.

The study of the rapid crack propagation in highly heterogeneous polymer materials is not trivial. Mechanisms of micro-cracking, micro- and macro-branching are described in the literature [16] [20] [21] [22]. The micro-branches which appear at a critical velocity and in connection with heterogeneities generate a complex roughness [23]. The crystalline phase (i.e. the spherulites) considerably disrupts the cracking path during rapid propagation. This seems to be at the ori- 
gin, together with the high cracking speed, of the branching mechanisms. In the literature, the effect of temperature on the dynamic behaviour of the fracture is, of which the authors are aware, not described for polymer-like materials. It is also relevant to note that all studies on the behaviour of rapid crack propagation have been carried out with elastic and brittle polymers and that the viscous effect has not been taken into account.

In this work, the main objective is to study the influence of temperature on the resistance to rapid crack propagation but also to initiate a first discussion on the role of viscosity and its link with a heterogeneous microstructure such as that of semi-crystalline materials. Tests on pipes are carried out. This geometry and semicrystallines are widely used for the transport of a fluid under pressure, hence the interest in this geometry [24] [25] [26]. The experimental campaigns carried out are described. The crack velocity and the fracture energy are estimated. Fracture surfaces are analyzed. The effect of temperature on fracture behaviour is finally discussed.

\section{Material and Methods}

\subsection{Material}

The material studied is the polyamide 11 (PA11), the Rilsan BESNO TL grade supplied by Arkema. The monomer is a castor oil derivative. It is therefore considered as a semi-crystalline bio-based polymer. Its glass transition temperature $T_{g}$ is of the order of $-50^{\circ} \mathrm{C}$. The material is therefore in a glassy state at room temperature. The degree of crystallinity of the polymer is $22 \%$. It was measured by differential scanning calorimetry. The pipe-shaped specimens were extruded. Pipe dimensions are: length $L=300 \mathrm{~mm}$, thickness $T=4 \mathrm{~mm}$, inner radius $R_{i}=$ $21 \mathrm{~mm}$ and the notch length $l_{n} \approx 5 R_{e} \approx 120 \mathrm{~mm}$ with $R_{e}$ the outer radius of the pipe. The notch length is defined with the help of numerical results. Indeed, the length of the notch is set to aim at a quasi-permanent regime of rapid propagation. In other words, a length for which the energy release rate reaches a plateau value and for which border effects are negligible. The pipes were kept in an airtight environment until the fracture test to limit the effects of humidity. The dynamic modulus $E_{d}$ was obtained by measuring the average ultrasonic velocity in the material $\left\langle v_{u}\right\rangle$ which is equal to $2100 \pm 46 \mathrm{~m} \cdot \mathrm{s}^{-1}$. The value $E_{d}=1620 \pm 82$ $\mathrm{MPa}$ is obtained from the Equation (1), where $v=0.43$ and $\rho=1040 \mathrm{~kg} \cdot \mathrm{m}^{-3}$. For the estimation of the fracture energy in dynamic propagation regime, only $E_{d}$ and $v$ will be considered to describe the behaviour of the material [27].

$$
E_{d}=\rho v_{u}^{2} \frac{(1+v)(1-2 v)}{1-v}
$$

\subsection{Dynamic Fracture Test}

Based on the latest studies [17] [28], a specific experimental device was used (see Figure 1). The pre-stress pipe specimen (PS2) is prestressed beforehand with the loading system being introduced into the pipe. It is then cracked using an 


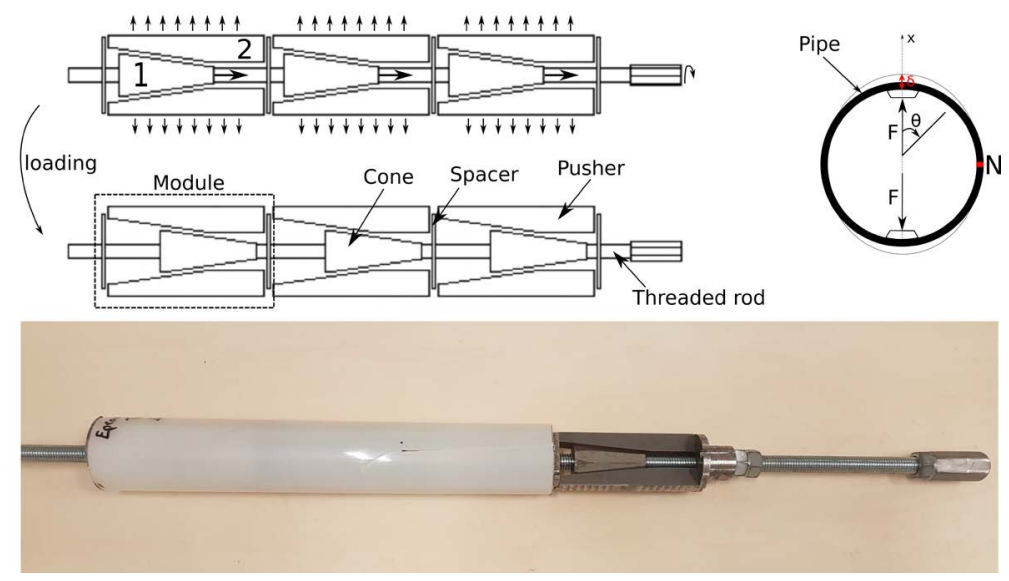

Figure 1. Loading system of PS2.

external impact. This system increases the elastic strain energy of the pipe by means of a mechanical device controlled in displacement: the relative internal displacement $(\delta)$ of the pipe. The loading system is a combination of three modules in series separated by spacers. Once threaded, the loading system is constrained by friction between the inner wall of the pipe and the metal parts. The overall rotation of the loading system is therefore not possible. The loading system with the pipe is then fixed to a frame. The rotation of a threaded rod by the operator will result in the displacement of steel cones. These cones are in contact with metal pushers that follow the internal surface of the pipe. These metal pushers are moved towards the outside of the pipe by the movement of the cones. Once the desired elastic strain energy has been reached, the internal displacement of the pipe is maintained (by stopping the rotation of the threaded rod). It should be noted that the displacement is imposed on $18 \%$ of the circumference of the pipe at two opposite poles. This necessarily induces bending at the two perpendicular poles. A mode I breakage test can nevertheless be considered. A relaxation time of the specimen (approximately 15 times the pre-stress time of the specimen) is considered before crack initiation. Crack initiation is then generated by the impact of a mass on a razor blade in contact with the crack tip. The notch is positioned at $\theta=-\frac{\pi}{2}$ with respect to the pushers.

\subsubsection{Temperature Conditioning of the Pipe}

The test is carried out at different temperatures. The pipe is cooled in a climatic chamber. The device (pipe + loading system) is placed inside the climatic chamber until the desired temperature is reached. Test temperatures range from $-20^{\circ} \mathrm{C}$ to room temperature. The temperature is measured just before crack initiation. Several readings are taken with a laser thermometer at different points along the crack propagation direction. The crack is initiated less than 30 seconds after leaving the climatic chamber of the device.

\subsubsection{Crack Tip Measurement}

When the crack propagates in dynamic regime, the spatio-temporal data of the 
crack tip is measured with a high-speed camera (Photron 106 APX-SA5) at 40,000 or 50,000 images per second with a resolution of $704 \times 256$ or $512 \times 272$ respectively. The quality of the recorded image was optimized by depositing a green paint on the sample surface. The thickness of the deposited paint is negligible and does not interfere with the fracture behavior of the pipe. Comparative tests with and without paint under the same loading conditions were carried out to validate this claim.

\subsubsection{Estimation of the Energy Release Rate}

The quasi-static energy release rate $G_{I 0}$ can be estimated analytically using the Equation (2) or numerically with a global energy balance [17]. The analytical estimation is based on the dynamometric ring model from the beam theory [17]. For the numerical model explained here [17], the fracture criterion considered is the average velocity $\dot{a}$ of the crack. This average velocity is measured using experimental data on the position of the crack tip as a function of time $a(t)$. The numerical method was validated by comparing $G_{I 0}$ obtained analytically with that obtained numerically. The dynamic energy release rate $G_{I D}$ is then estimated by taking into account the inertia effects that vary as a function of the crack velocity and the geometry of the pipe. $G_{I 0}$ depends on the value of the Young's modulus $E$ of the material. It is well established that $E$ varies as a function of temperature. According to [29], for a polyamide subjected to quasi-static tensile stress, a decrease in temperature of $20^{\circ} \mathrm{C}-40^{\circ} \mathrm{C}$ (relative to room temperature) induces an increase in Young's modulus of 5\%. To estimate $G_{I 0}$ the effect of temperature on $E$ was considered. On the other hand, the dynamic Young's modulus $E_{d}$ does not vary whatever the test temperature (result obtained by ultrasonic measurement).

$$
G_{I 0}=\frac{8 \pi E\left(R_{e}-R_{i}\right)^{2} \delta^{2}}{3\left(\pi^{2}-8\right)\left(R_{e}+R_{i}\right)^{3}}
$$

$R_{e}$ and $R_{i}$ are the outer and inner radii of the pipe, respectively.

The dynamic correction $k$ to consider to estimate $G_{I D}=k G_{I 0}$ is worth about 0.75 to $0.6 c_{R}$. The Rayleigh wave velocity $\left(c_{R}\right)$ in PA11 is about $698 \mathrm{~m} \cdot \mathrm{s}^{-1}$ [17]. In other words, $75 \%$ of the energy is dissipated by the structure as inertia effects when the crack propagates at $60 \%$ of the Rayleigh wave velocity in the material in the case of PS2 geometry. Note that the value of Young's modulus does not influence the value of $k$.

Two cracking configurations are shown in Figure 2 and Figure 3: a single crack propagation perpendicular to the loading axis (see Figure 2) and a main crack propagation that branches into several secondary cracks (see Figure 3 ). In dynamic regime and for this type of multiphase materials with strong heterogeneities, the energy is mainly dissipated by surface creation. An increase in the energy release rate and/or a decrease in temperature can induce in fine macroscopic crack branching. The energy release rate can only be estimated when a longitudinal crack propagates. 


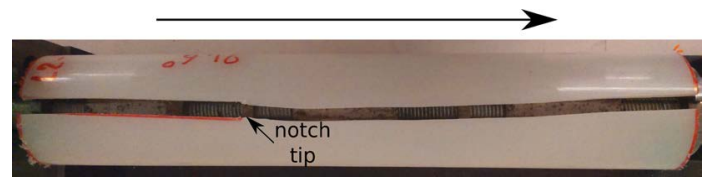

Figure 2. Capture of a pipe and the loading system showing a single longitudinal crack (sample PS2-32). The arrow indicates the direction of propagation.

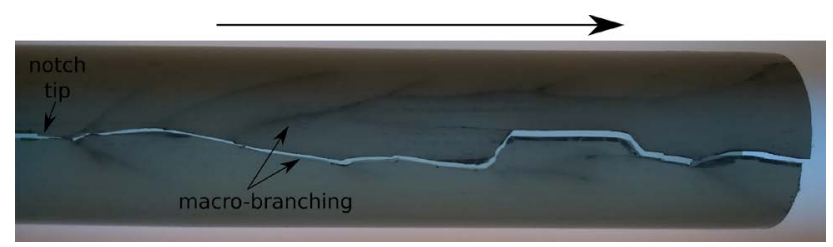

Figure 3. Capture of a pipe with many branches. The arrow indicates the direction of propagation.

\subsection{Analysis of the Fracture Surface}

A Zeiss EVOHD 15 scanning electron microscope (SEM) was used to observe the fracture surface of the samples. Samples were taken from the cracked pipes. They are embedded in acrylic resin and metallized in order to visualize them on the SEM. The apparatus used for metallization is a Cressington 108 auto. The gold layer is assumed to be of constant thickness on the surface of the sample. An accelerated voltage of $10 \mathrm{keV}$ is used with a current of $200 \mathrm{pA}$.

\section{Results}

The influence of the temperature on the fracture behaviour was studied. Two types of tests were carried out and correspond to the two next sub-sections:

1) The relative radial displacement $\delta$ was adjusted (i.e. determined experimentally) as a function of temperature to limit the stored elastic energy and induce only one longitudinal macro-crack whatever the temperature is;

2) The relative displacement was kept constant for the different temperatures' tests. Its value was set such that it generates a lonely longitudinal crack at room temperature.

The results of these tests, which are given in Table 1, are described in the following paragraphs. The values of $G_{I D}$ were calculated for a single crack by considering $k=0.75$ obtained for an average speed of about $0.6 c_{R}$. For samples PS2-19 and PS2-27 a value of $G_{I D}$ is given because the branching took place only a few centimetres before the end of the pipe.

\subsection{Temperature Influence for Single Crack Testing}

For these tests, the propagation of a longitudinal macro-crack was analysed. The spatio-temporal data of the crack tip was measured. The fracture energy was estimated. And the fracture surface was qualitatively described.

\subsubsection{Estimation of Crack Velocity}

The spatio-temporal data of the crack tip was recorded by high-speed camera 
Table 1. Raw data from the two test campaigns. The sample name, the fracture energy $G_{I D}\left(\mathrm{~kJ} \cdot \mathrm{m}^{-2}\right)$, the crack velocity $\dot{a}\left(\mathrm{~m} \cdot \mathrm{s}^{-1}\right)$, the temperature $T\left({ }^{\circ} \mathrm{C}\right)$ and the crack length $\delta a(\mathrm{~mm})$ are referenced. It is also indicated whether or not the main crack is macro-branched or not (yes or no).

\begin{tabular}{|c|c|c|c|c|c|}
\hline Sample & $T\left({ }^{\circ} \mathrm{C}\right)$ & $\dot{a}$ & branching? & $G_{I D}$ & $\delta a$ \\
\hline PS2-1 & -16 & 456 & no & 1.1 & 200 \\
\hline PS2-2 & -15 & 430 & no & 1.3 & 201 \\
\hline PS2-3 & -18 & 377 & no & 1.2 & 202 \\
\hline PS2-4 & -17 & 412 & no & 2.1 & 198 \\
\hline PS2-5 & -10 & 426 & no & 1.0 & 199 \\
\hline PS2-6 & -15 & 466 & no & 1.8 & 200 \\
\hline PS2-7 & -15 & 457 & no & 2.2 & 200 \\
\hline PS2-8 & -12 & 436 & no & 1.3 & 199 \\
\hline PS2-9 & -14 & 424 & no & 0.9 & 201 \\
\hline PS2-10 & -18 & 412 & no & 1.2 & 200 \\
\hline PS2-11 & -4.8 & 421 & yes & NA & 418 \\
\hline PS2-12 & -5.6 & 432 & yes & NA & 449 \\
\hline PS2-13 & -5.3 & 398 & yes & NA & 655 \\
\hline PS2-14 & -0.9 & 457 & yes & NA & 347 \\
\hline PS2-15 & -0.5 & 425 & yes & NA & 298 \\
\hline PS2-16 & 0.4 & 436 & yes & NA & 273 \\
\hline PS2-17 & 9.4 & 426 & no & 1.5 & 220 \\
\hline PS2-18 & 9.8 & 462 & no & 1.5 & 193 \\
\hline PS2-19 & 9.5 & 435 & yes & NA & 281 \\
\hline PS2-20 & 12.4 & 426 & no & 1.4 & 201 \\
\hline PS2-21 & 14.6 & 460 & no & 1.3 & 204 \\
\hline PS2-22 & 15.7 & 377 & no & 1.3 & 202 \\
\hline PS2-23 & 17 & 395 & no & 1.4 & 200 \\
\hline PS2-24 & 17.2 & 390 & no & 1.6 & 201 \\
\hline PS2-25 & 18.5 & 405 & no & 2.0 & 198 \\
\hline PS2-26 & 20.5 & 400 & no & 1.3 & 200 \\
\hline PS2-27 & 20.7 & 426 & yes & NA & 244 \\
\hline PS2-28 & 20.4 & 435 & no & 1.4 & 200 \\
\hline PS2-29 & 20.8 & 405 & no & 1.8 & 204 \\
\hline PS2-30 & 18.6 & 400 & no & 1.6 & 200 \\
\hline PS2-31 & 20 & 412 & no & 1.7 & 199 \\
\hline PS2-32 & 21 & 457 & no & 2.0 & 205 \\
\hline PS2-33 & 21 & 441 & no & 2.1 & 200 \\
\hline
\end{tabular}


(see Figure 4). In the image 1, the crack is initiated in dynamic regime. The crack continues its propagation as can be seen in the following images. It ends up passing completely through the pipe (image 6). The tracking of the crack tip is facilitated by the green paint deposited on the surface of the sample. This paint fragments as the crack progresses. For this test, performed at about $-10^{\circ} \mathrm{C}$, one can see that the crack propagates perpendicular to the pipe stress direction without generating macrobranching.

In a fast propagating regime, the velocity of the macroscopic crack tip appears constant regardless of $G_{10}$ [17]. Here, the average cracking velocity was expressed as a function of temperature (Figure 5). It can be observed here that the macroscopic velocity of the crack tip is also constant whatever the test temperature is, and is around $420 \mathrm{~m} \cdot \mathrm{s}^{-1}$.

\subsubsection{Estimation of Fracture Energy}

The fracture energy $G_{I D}$ was estimated using a numerical model where inertia effects were taken into account. The evolution of $G_{I D}$ as a function of temperature is shown in Figure 6. One can see that for a given temperature, the value of $G_{I D}$ varies significantly. At room temperature for example, the fracture energy varies from simple to double. The scatter in the value of $G_{I D}$ is known from the literature and can be associated with surface roughness [30] [31]. For a rubber

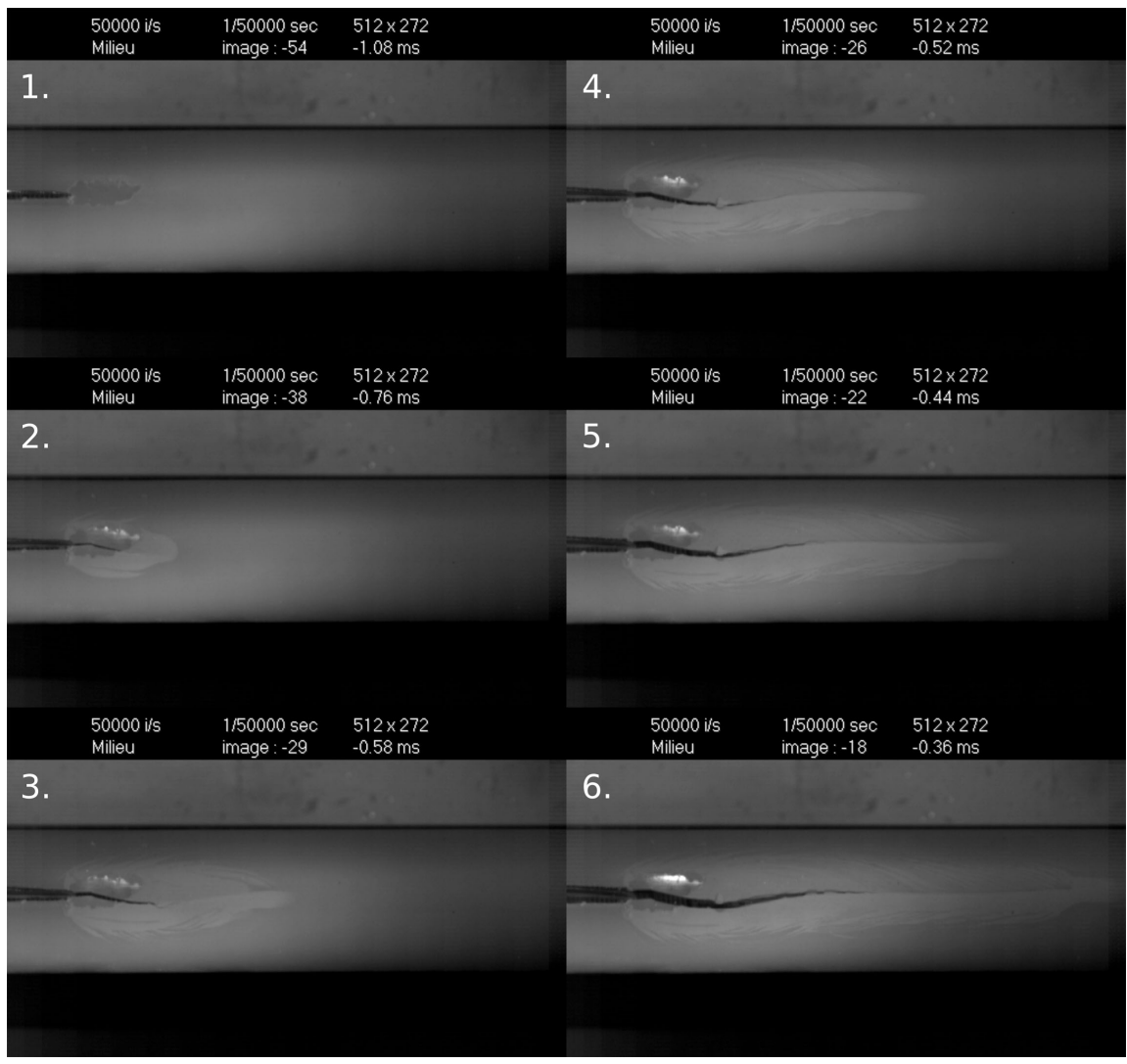

Figure 4. Fast camera images with a sampling rate of 50,000 frames per second to visualize the rapid propagation of the crack in the PS2-5 specimen. The direction of propagation is from left to right. 


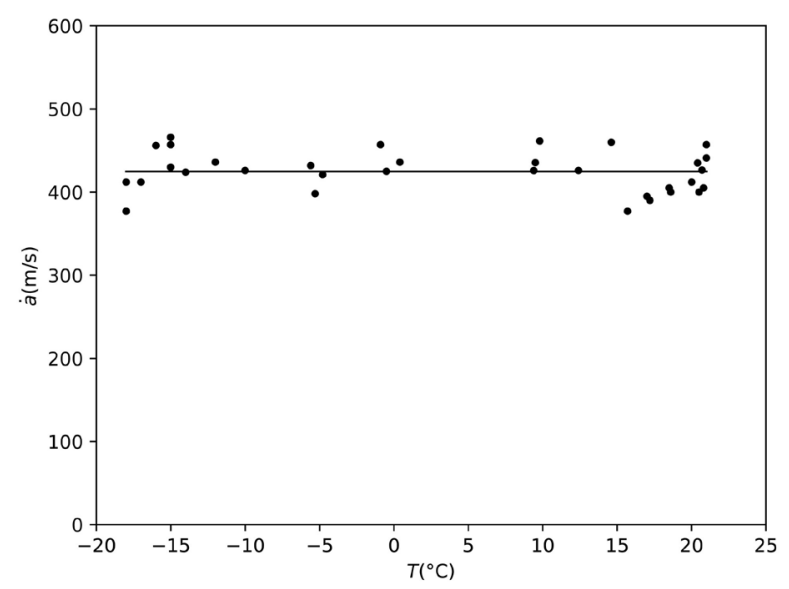

Figure 5. Evolution of the average velocity of the macroscopic crack tip as a function of temperature for all tests.

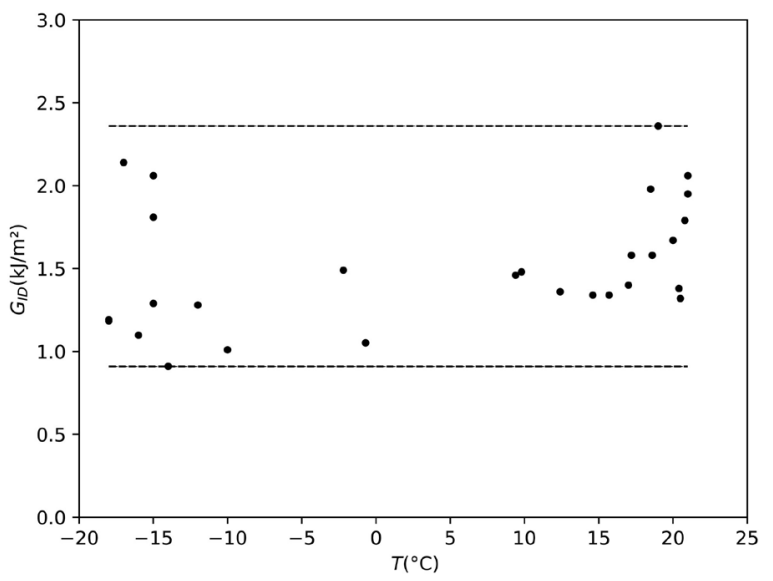

Figure 6. Evolution of the fracture energy as a function of temperature.

toughened (RT) PMMA, it has been shown that the scatter in the value of $G_{I D}$ and the amount of surface created were of the same order of magnitude. The heterogeneities of the microstructure are comparable between a RT-PMMA and a semicrystalline. They are sources of instabilities generating more or less rough surfaces. It is therefore reasonable to be convinced that the source of this scatter is the surface roughness, which is not taken into account in the estimation of $G$.

When we plot the evolution of the fracture energy as a function of crack velocity and temperature (for the lowest temperatures $-15^{\circ} \mathrm{C}$ and the highest $20^{\circ} \mathrm{C}$ ), this scatter is confirmed (see in Figure 7). Nevertheless, it can be seen that there does not seem to be a major effect of the test temperature on the mean value of the fracture energy. Indeed, the scatter in the value of $G_{I D}$ is similar to $-15^{\circ} \mathrm{C}$ and $20^{\circ} \mathrm{C}$.

\subsubsection{Analysis of Fracture Surfaces}

The analysis of the fracture surfaces can be very instructive [21] [22] [30] [31] [32] [33]. Initiation and propagation zones were observed. In the initiation zone (see Figure 8 ) there is a macroscopically smooth area just after the razor blade 


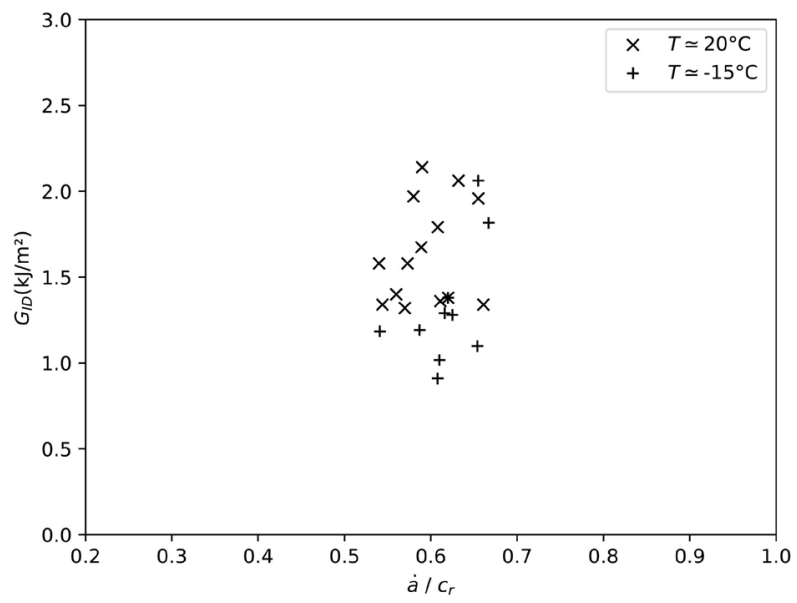

Figure 7. Evolution of the fracture energy as a function of the propagation velocity of the crack normalized with respect to $c_{R}$ for the minimum and maximum temperatures.

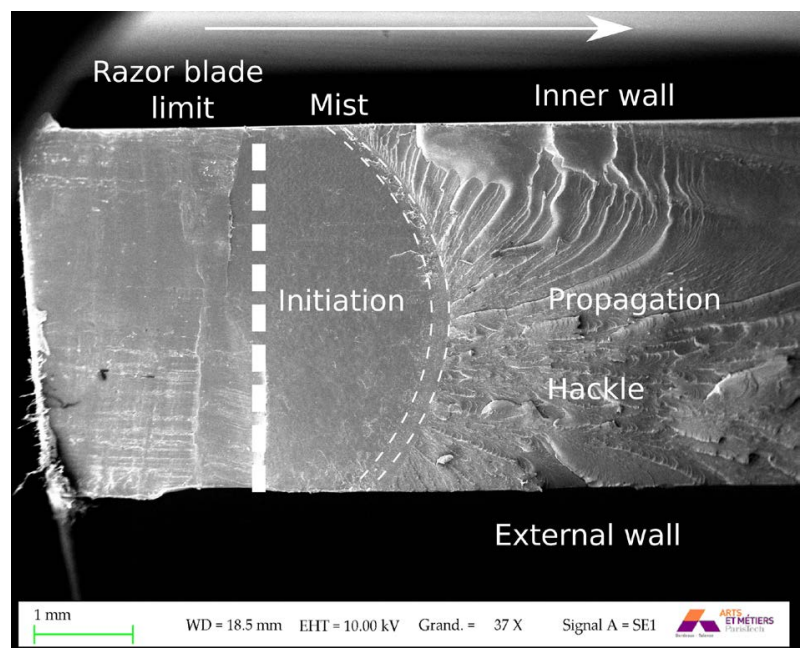

Figure 8. Electron microscopy observation of the initiation zone of the fracture surface of the SBS-30 sample. The arrow indicates the direction of propagation.

mark. An intermediate zone ("mist") is visible at the end of this smooth zone. In this zone, we observe an arrest mark (i.e. a "rib" marking) of the main crack and a plastic deformation of the matrix, the origin of which is discussed in [16]. The propagation zone is more chaotic ("hackle" zone) in terms of apparent roughness. The surface is rough and relatively complex to analyze. One can also notice the influence of the external loading on the roughness in the thickness of the sample. It varies as the energy release rate due to the bending of the pipe wall at this level of the sample. The energy release rate is greater towards the outside of the pipe than towards the inside.

Regardless of the test temperature, comparable observations were made. There did not appear to be any significant difference in surface roughness as a function of temperature. On the other hand, the energy release rate has a strong influence on surface roughness. In the thickness already we notice that the larger the radius (and thus $G$ ) the rougher the surface is. On the other hand, the greater 
the elastic strain energy stored in the pipe, the rougher the surface will be.

Two types of surface roughness are shown in Figure 9 and Figure 10 for the samples PS2-33 and PS2-19 being propagated. The elastic strain energy stored in the pipe before impact (and therefore the fracture energy $G_{I D}$ ) is significantly greater for sample PS2-33 than PS2-19. For sample PS2-33, the roughness is significant towards the outside of the pipe as opposed to sample PS2-19.

\subsection{Temperature Influence for Multi-Crack Testing}

In order to study the influence of temperature on the fracture behaviour of polymer pipes, another test configuration was considered. These tests were all performed at internal iso-displacement $\delta$. The elastic strain energy stored in the pipe is not strictly identical because the Young's modulus varies as a function of temperature. Nevertheless being below the glass transition temperature (about

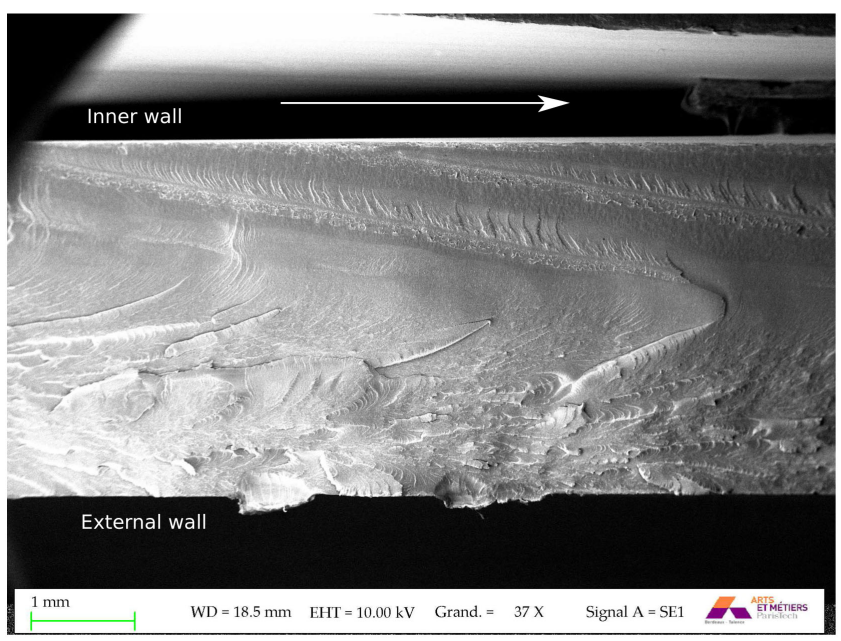

Figure 9. Observation by electron microscopy of the propagation zone of the fracture surface of sample PS2-33. The arrow indicates the direction of propagation.

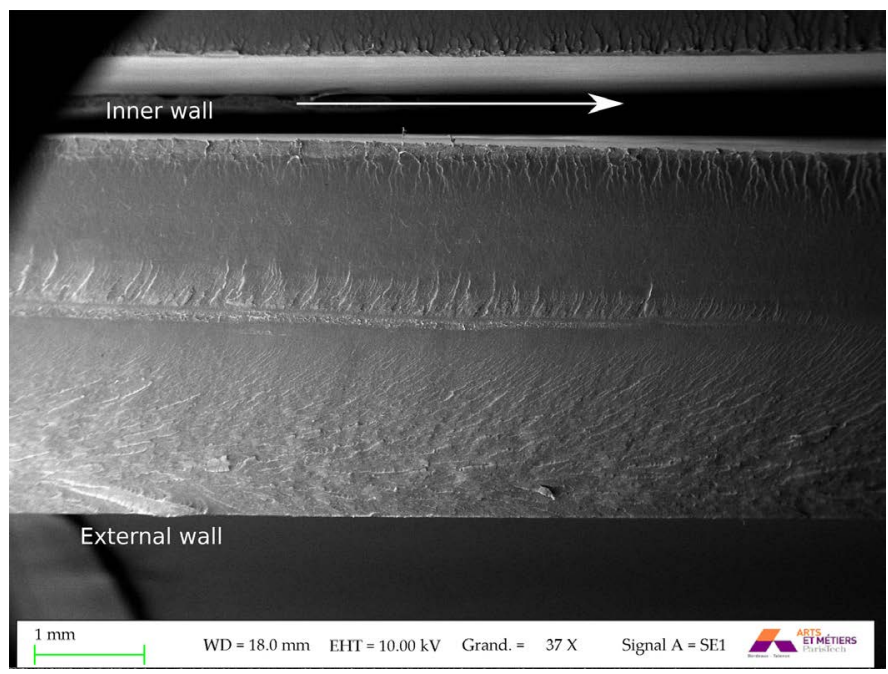

Figure 10. Observation by electron microscopy of the propagation zone of the fracture surface of sample PS2-19. The arrow indicates the direction of propagation. 
$50^{\circ} \mathrm{C}$ ) the material is in a glassy state. Its viscoelastic behavior therefore varies slightly between room temperature and $-5^{\circ} \mathrm{C}$. The Young's modulus of the material at $-5^{\circ} \mathrm{C}$ is about $5 \%$ higher than at room temperature. The minimum test temperature was set at $-5^{\circ} \mathrm{C}$ because below this temperature the number of observed macrobranches is considerable (see Figure 3 ).

\subsubsection{Estimation of Crack Velocity}

The position of the crack tip as a function of time was recorded using the fast camera (see Figure 11). The beginning of the propagation is visible on the image 1. A macroscopic branching is then initiated (see image 2). The branches continue their propagation at the same velocity and in symmetrical directions with respect to the main cracking axis (Images 3 to 5). The cracks eventually open the pipe completely. Debris is generated by the branching mechanism of the main crack. Due to inertia effects, this debris is ejected from the pipe (Image 6).

The crack velocity was calculated before and after the macroscopic branching of the crack (see Figure 12). The macroscopic velocity of the branches appears to be identical to that of the main crack. The mean cracking velocities estimated for the branched cracks are of the order of $0.6 c_{R}$ (see Figure 5). Whether the main crack is branching or not, the macroscopic velocity of the crack appears to be of the same order of magnitude.

\subsubsection{Calculation of Crack Lengths}

Since it was difficult to assess the fracture energy of the material when the crack branches, the total projected length of the cracks $(\delta a)$ was measured using a ruler. The evolution of $\delta a$ as a function of temperature is shown in Figure 13. At internal iso-displacement, the more the temperature decreases, the greater the $\delta a$. The minimum length of a longitudinal crack is about $200 \mathrm{~mm}$. At $-5^{\circ} \mathrm{C}$ this

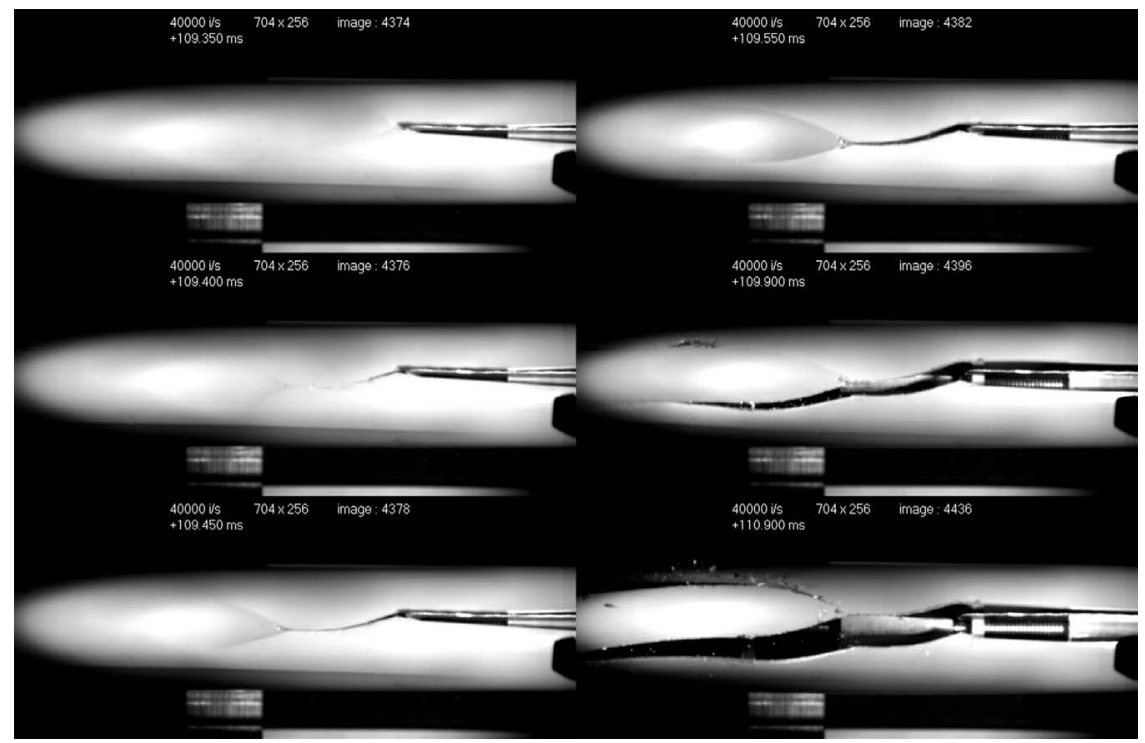

Figure 11. Fast camera images with a sampling rate of 40,000 frames per second to visualize the rapid crack propagation of the PS2-14 specimen. The direction of propagation is from right to left. 


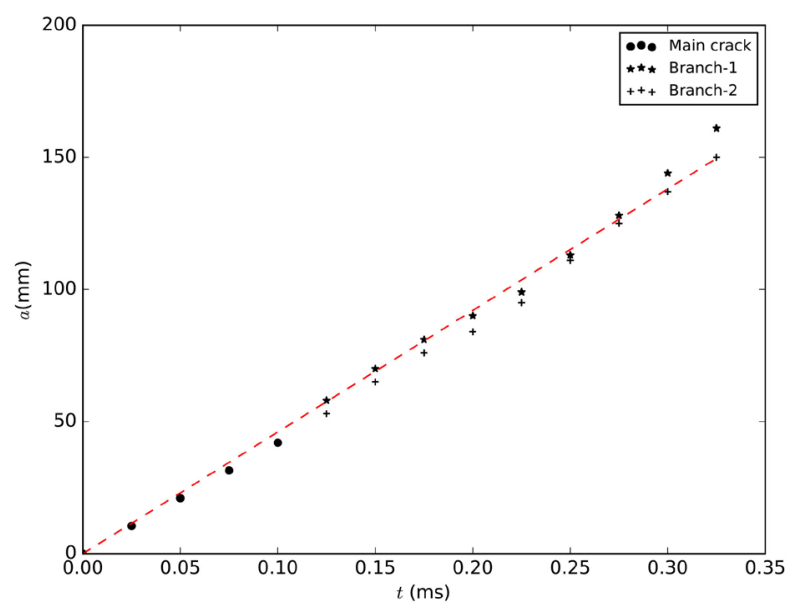

Figure 12. Evolution of $a(t)$ before and after branching for sample PS2-16.

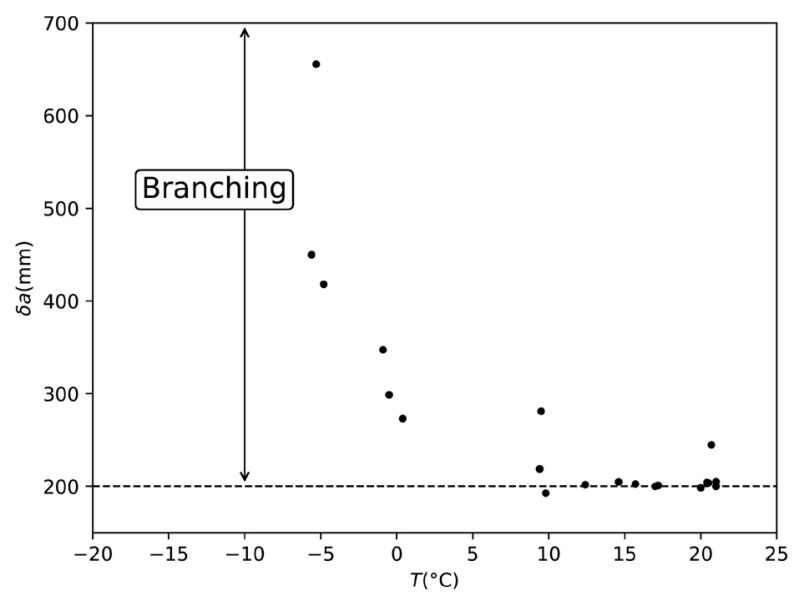

Figure 13. Evolution of total crack length $\delta a$ as a function of temperature.

length is multiplied by a coefficient between 2 and 3.5. This seems to confirm the increase in the number of macroscopic branches with a decrease in temperature.

\section{Discussion}

The single crack tests allowed us, by applying the LEFM formalism, to estimate the fracture energy as a function of crack velocity and temperature. The spatio-temporal data of the crack tip informs us that the velocity of the macroscopic crack front does not seem to be influenced by the test temperature. In the temperature range tested, the material is in a glassy state. No major transitions (e.g. $T_{g}$ ) are exceeded. The fracture energy has been estimated. Depending on the crack velocity, $G_{I D}$ appears to be non-unique and varies from single to more than double for the lowest $\left(\approx-15^{\circ} \mathrm{C}\right)$ and highest $\left(\approx 20^{\circ} \mathrm{C}\right)$ temperatures. This is not associated with temperature, which does not appear to have a major effect on the fracture energy. This can be explained by the time-temperature equivalence for visco-elastic polymer materials. The temperature variation is not significant $\left(\Delta T \approx 35^{\circ} \mathrm{C}\right)$. On the contrary, the crack velocity $\left(\approx 0.6 c_{R}\right)$. The effect of the 
strain rate induced by the rapid crack propagation seems to be of the first order compared to the effect of the temperature decrease. In a dynamic propagation regime, a decrease in temperature of a few tens of degrees does not seem to have any effect on crack velocity and fracture energy.

For crack initiation resistance it seems to be different. It was found that less impact energy was required to initiate the crack at cold temperature than at room temperature. Temperature appears to have a significant effect on crack initiation resistance. This is due to the quasi-static loading during preloading. A decrease in temperature makes a semi-crystalline material more brittle. Its resistance to crack initiation is therefore reduced. This can be explained by the limitation of macromolecular mobility. Locally the mechanisms of cavitation damage and plastic flow, visible before the crack propagation in quasi-static regime, are limited. Crack initiation resistance tests with cyclic tests and a "compact tension" geometry will make it possible to quantify this influence and the difference in deviation from the resistance to the rapid propagation of a crack.

The fracture surfaces were analysed using a SEM. There does not appear to be a significant effect of temperature on surface roughness over the temperature range studied. The effect of temperature appears to be of the second order compared to the effect of the energy release rate. The higher the energy release rate, the rougher the surface. The variability highlighted on the value of the fracture energy at $0.6 c_{R}$ seems to be largely explained by the difference in surface roughness. A potentially large surface roughness can generate a large amount of surfacing created. This is not taken into account in the estimation of the fracture energy, which considers the quantity of projected surface (the thickness of the sample times the length of the crack) [30] [31].

Internal iso-displacement tests were carried out at different temperatures. Macro-branching mechanisms appeared regularly and in large numbers around $-5^{\circ} \mathrm{C}$. Crack velocities were calculated before and after macro-branching. It seems to be equal $\left(\approx 0.6 c_{R}\right)$. This confirms that the temperature does not seem to have any effect on the macroscopic velocity of the propagating crack, whether there is branching of the main crack or not. The total crack length was estimated. A significant increase in this magnitude with a decrease in temperature was demonstrated. This reflects a significant increase in the probability of branching the main crack with a decrease in temperature.

At room temperature, tests on the same material but for a different geometry (strip band specimen) seem to confirm the results obtained on 1) the crack velocity, 2) the link between the energy release rate and surface roughness and 3) the variability on the value of the fracture energy at $0.6 c_{R}$ [16]. It has been postulated that at room temperature the main crack appears to dissipate more energy with micro-branching than with macro-branching [16]. In this study, internal iso-displacement testing revealed a high proportion of specimens that generated macro-branching mechanisms at $-5^{\circ} \mathrm{C}$. As temperature decreases the amorphous phase of the material becomes more brittle. The crystalline phase of a semi-crystalline material is already considered brittle at room temperature. This 
is confirmed by the ease of cold crack initiation. In quasi-static state, the amorphous phase is ductile at room temperature and becomes brittle when cold. The volume of the amorphous phase is considerable (about $80 \%$ ). If one considers that there is no longer a significant difference in fracture behaviour between the amorphous and crystalline phases when cold, then the defects of the amorphous phase become potentially as critical as the defects of the crystalline phase (i.e. spherolites). It has been shown in a previous article that during propagation the crack seems to bifurcate and micro-branch at the level of the spherolites [16]. By decreasing the temperature, amorphous phase defects potentially larger than spherulites (of the order of $10-20 \mu \mathrm{m}$ ) can interact in the cracking path. Macro-branching mechanisms are generated.

\section{Conclusion}

The influence of temperature on the resistance to rapid crack propagation has been studied for a semi-crystalline bio-based polymer material: PA11. Two types of tests were carried out: tests with a single longitudinal crack and others with internal pipe iso-displacement. The first tests show that a decrease in temperature does not seem to have a significant effect on crack propagation velocity, surface roughness and fracture energy variability at $0.6 c_{R}$. For the second type of tests, a decrease in temperature increases the probability of macroscopic branching of the main crack. The difference in fracture behaviour between the amorphous and crystalline phase varies significantly as a function of temperature. The difference between the resistance to initiation and rapid propagation also varies. Although temperature does not seem to have a major impact on the estimated parameters, it does seem to have an impact on the difference between the resistance to initiation and the resistance to rapid propagation. This difference seems to be significantly reduced by lowering the temperature. The mechanisms of cavitation damage and plastic flow are increasingly limited by the decrease in temperature (and therefore in macromolecular mobility). Cracking in the pipe is more extensive and therefore more critical. This is emphasised in particular by the probability of the material to be macro-branched as the temperature decreases. The proportion of defects or heterogeneities likely to generate a macrobranch is increased when the amorphous phase sees its macromolecular mobility limited by a decrease in temperature. Being more brittle, the amorphous phase becomes an important source of crack initiation sites. A possible avenue of study for this work would be to study the influence of temperature on the initiation resistance of the material in order to evaluate the deviation from the propagation resistance. For taking into account the surface roughness and the fine evaluation of the fracture energy during rapid propagation for this type of multiphase material (amorphous and crystalline phases), a specific rapid cracking setup is necessary. Its principle is already described in [16].

\section{Acknowledgements}

The authors would like to warmly thank the participation in this work of Valen- 
tin Honno and Annouck Bradu who did a Bachelor internship on the subject and Jérémie Béga for his technical support on microscopic analyses.

\section{Conflicts of Interest}

The authors declare no conflicts of interest regarding the publication of this paper.

\section{References}

[1] Schultz, J. (1984) Microstructural Aspects of Failure in Semicrystalline Polymers. Polymer Engineering and Science, 24, 770-785.

https://doi.org/10.1002/pen.760241007

[2] Cotterell, B., Chia, J. and Hbaieb, K. (2007) Fracture Mechanisms and Fracture Toughness in Semicrystalline Polymer Nanocomposites. Engineering Fracture Mechanics, 74, 1054-1078. https://doi.org/10.1016/j.engfracmech.2006.12.023

[3] Rolland, H., Saintier, N., Raphael, I., Lenoir, N., King, A. and Robert, G. (2018) Fatigue Damage Mechanisms of Short Fiber Reinforced pa66 as Observed by In-Situ Synchrotron X-Ray Microtomography. Composites Part B: Engineering, 143, 217-229. https://doi.org/10.1016/j.compositesb.2017.12.051

[4] Bles, G., Nowacki, W. and Tourabi, A. (2009) Experimental Study of the Cyclic Visco-Elasto-Plastic Behaviour of a Polyamide Fibre Strap. International Journal of Solids and Structures, 46, 2693-2705. https://doi.org/10.1016/j.ijsolstr.2009.02.015

[5] Poulet, P., Hochstetter, G., King, A., Proudhon, H., Joannès, S. and Laiarinandrasana, L. (2016) Observations by In-Situ X-Ray Synchrotron Computed Tomography of the Microstructural Evolution of Semi-Crystalline Polyamide 11 during Deformation. Polymer Testing, 56, 245-260.

https://doi.org/10.1016/j.polymertesting.2016.10.023

[6] Cayzac, H.A., Saï, K. and Laiarinandrasana, L. (2013) Damage Based Constitutive Relationships in Semi-Crystalline Polymer by Using Multi-Mechanisms Model. International Journal of Plasticity, 51, 47-64. https://doi.org/10.1016/j.ijplas.2013.06.008

[7] Boisot, G., Laiarinandrasana, L., Besson, J., Fond, C. and Hochstetter, G. (2011) Experimental Investigations and Modeling of Volume Change Induced by Void Growth in Polyamide 11. International Journal of Solids and Structures, 48, 2642-2654. https://doi.org/10.1016/j.ijsolstr.2011.05.016

[8] Castagnet, S., Gacougnolle, J.L. and Dang, P. (2000) Correlation between Macroscopical Viscoelastic Behaviour and Micromechanisms in Strained $\alpha$ Polyvinylidene Fluoride (PVDF). Materials Science and Engineering. A, 276, 152-159. https://doi.org/10.1016/S0921-5093(99)00320-2

[9] Selles, N., Cloetens, P., Proudhon, H., Morgeneyer, T.F., Klinkova, O., Saintier, N. and Laiarinandrasana, L. (2017) Voiding Mechanisms in Deformed Polyamide 6 Observed at the Nanometric Scale. Macromolecules, 50, 4372-4383. https://doi.org/10.1021/acs.macromol.7b00727

[10] Zhurkov, S.N., Zakrevskyi, V.A., Korsukov, V.E. and Kuksenko, V.S. (1972) Mechanism of Submicrocrack Generation in Stressed Polymers. Journal of Polymer Science Part A-2: Polymer Physics, 10, 1509-1520. https://doi.org/10.1002/pol.1972.160100808

[11] Zhurkov, S.N. and Kuksenko, V.S. (1875) The Micromechanics of Polymer Fracture. International Journal of Fracture, 11, 629-639. 
https://doi.org/10.1007/BF00116370

[12] Séguéla, R. and Darras, O. (1994) Phenomenological Aspects of the Double Yield of Polyethylene and Related Copolymers under Tensile Loading. Journal of Materials Science, 29, 5342-5352. https://doi.org/10.1007/BF01171546

[13] Maïza, S., Lefebvre, X., Brusselle-Dupend, N., Klopffer, M.H., Cangémi, L., Castagnet, S. and Grandidier, J. (2019) Physicochemical and Mechanical Degradation of Polyamide 11 Induced by Hydrolysis and Thermal Aging. Journal of Applied Polymer Science, 136, 47,628. https://doi.org/10.1002/app.47628

[14] Humbert, S., Lame, O. and Vigier, G. (2009) Polyethylene Yielding Behaviour: What Is behind the Correlation between Yield Stress and Crystallinity? Polymer, 50, 3755-3761. https://doi.org/10.1016/j.polymer.2009.05.017

[15] Darras, O. and Séguéla, R. (1993) Tensile Yield of Polyethylene in Relation to Crystal Thickness. Journal of Polymer Science Part B: Polymer Physics, 31, 759-766. https://doi.org/10.1002/polb.1993.090310702

[16] Kopp, J.B. and Girardot, J. (2020) Dynamic Fracture in a Semicrystalline Biobased Polymer: An Analysis of the Fracture Surface. International Journal of Fracture, 226, 121-132. https://doi.org/10.1007/s10704-020-00482-y

[17] Kopp, J.B., Fond, C. and Hochstetter, G. (2018) Rapid Crack Propagation in pa11: An Application to Pipe Structure. Engineering Fracture Mechanics, 202, 445-457. https://doi.org/10.1016/j.engfracmech.2018.08.025

[18] Messiha, M., Frank, A., Koch, T., Arbeiter, F. and Pinter, G. (2020) Effect of Polyethylene and Polypropylene Cross-Contamination on Slow Crack Growth Resistance. International Journal of Polymer Analysis and Characterization, 25, 649-666. https://doi.org/10.1080/1023666X.2020.1833143

[19] Messiha, M., Gerets, B., Heimink, J., Frank, A., Arbeiter, F. and Engelsing, K. (2020) Slow Crack Growth Resistance of Modern pa-u12 Grades Measured by Cyclic Cracked Round Bar Tests and Strain Hardening Tests. Polymer Testing, 86, 106,468. https://doi.org/10.1016/j.polymertesting.2020.106468

[20] Dalmas, D., Guerra, C., Scheibert, J. and Bonamy, D. (2013) Damage Mechanisms in the Dynamic Fracture of Nominally Brittle Polymers. International Journal of Fracture, 184, 93-111. https://doi.org/10.1007/s10704-013-9839-y

[21] Guerra Amaro, C.M. (2009) Dynamic Fracture in Brittle Amorphous Materials: Dissipation Mechanisms and Dynamically-Induced Microcracking in PMMA. Theses, Ecole Polytechnique X. https://pastel.archives-ouvertes.fr/pastel-00006135

[22] Arakawa, K. and Takahashi, K. (1991) Relationships between Fracture Parameters and Fracture Surface Roughness of Brittle Polymers. International Journal of Fracture, 48, 103-114. https://doi.org/10.1007/BF00018393

[23] Sharon, E. and Fineberg, J. (1996) Microbranching Instability and the Dynamic Fracture of Brittle Materials. Physical Review B-Condensed Matter and Materials Physics, 54, 7128-7139. https://doi.org/10.1103/PhysRevB.54.7128

[24] Mason, J. and Chen, J. (2006) Establishing the Correlation between s4 and Full Scale Rapid Crack Propagation Testing for Polyamide-11 (pa-11) Pipe. Plastic Pipes XIII.

[25] Williams, J. and Venizelos, G. (1999) A Perturbation Analysis of Rapid Crack Propagation in Pressurised Pipe. International Journal of Fracture, 94, 161-176.

[26] Greenshields, C., Venizelos, G. and Ivankovic, A. (2000) A Fluid-Structure Model for Fast Brittle Fracture in Plastic Pipes. Journal of Fluids and Structures, 14, 221-234. https://doi.org/10.1006/jfls.1999.0258

[27] Bradley, W., Cantwell, W. and Kausch, H. (1997) Viscoelastic Creep Crack Growth: 
A Review of Fracture Mechanical Analyses. Mechanics Time-Dependent Materials, 1, 241-268. https://doi.org/10.1023/A:1009766516429

[28] Kopp, J.B., Lin, J., Schmittbuhl, J. and Fond, C. (2014) Longitudinal Dynamic Fracture of Polymer Pipes. European Journal of Environmental and Civil Engineering, 18, 1097-1105.

[29] Guerin, B. (1994) Polyamides. Techniques de l'ingénieur. Plastiques et composites 2.

[30] Kopp, J.B., Schmittbuhl, J., Noel, O., Lin, J. and Fond, C. (2014) Fluctuations of the Dynamic Fracture Energy Values Related to the Amount of Created Fracture Surface. Engineering Fracture Mechanics, 126, 178-189.

https://doi.org/10.1016/j.engfracmech.2014.05.014

[31] Kopp, J.B., Schmittbuhl, J., Noel, O. and Fond, C. (2015) A Self-Affine Geometrical Model of Dynamic rt-pmma Fractures: Implications for Fracture Energy Measurements. International Journal of Fracture, 193, 141-152.

https://doi.org/10.1007/s10704-015-0025-2

[32] Fineberg, J. and Bouchbinder, E. (2015) Recent Developments in Dynamic Fracture: Some Perspectives. International Journal of Fracture, 196, 33-57.

https://doi.org/10.1007/s10704-015-0038-x

[33] Ravi-Chandar, K. and Knauss, W. (1984) An Experimental Investigation into Dynamic Fracture: II. Microstructural Aspects. International Journal of Fracture, 26, 65-80. https://doi.org/10.1007/BF01152313 